\title{
SELECCIÓN, MULTIPLICACIÓN Y EVALUACIÓN INICIAL DE CLONES DE Eucalyptus camaldulensis PARA LAS ZONAS ÁRIDAS Y SEMIÁRIDAS DE CHILE
}

\author{
Braulio Gutiérrez C. ${ }^{1}$ y Maria Paz Molina B. ${ }^{1}$
}

\section{RESUMEN}

Las zonas áridas y semiáridas de la zona central del pais se caracterizan por condiciones climáticas adversas que limitan el desarrollo productivo del sector silvoagropecuario. Diversos esfuerzos han sido efectuados para desarrollar alternativas productivas compatibles con tal limitación, identificándose entre ellos al cultivo de especies forestales resistentes a la sequia.

Entre tales especies, Eucalyptus camaldulensis ha demostrado una gran adaptación a condiciones de sequia, constituyendo en la actualidad una de las alternativas más conocidas para establecer cultivos forestales en estas zonas.

Al respecto, la gran extensión de la distribución natural de esta especie en su lugar de origen, le confiere una considerable variabilidad intraespecifica en distintos caracteres de interés, entre ellos la tolerancia a la sequia. Esta variabilidad sumada a la experiencia empirica obtenida en el establecimiento, manejo y evaluación de ensayos de $E$. camaldulensis en zonas áridas y semiáridas, permite reconocer la existencia de individuos con una tolerancia a condiciones de estrés hidrico superior al promedio, los cuales son capaces de exhibir interesantes tasas de crecimiento, aún bajo condiciones deficitarias de aporte hidrico.

Reconociendo los enunciados anteriores, en el presente articulo se entrega los principales resultados de un proyecto de investigación destinado a la masificación clonal de individuos de $E$. camaldulensis seleccionados en función de su crecimiento y resistencia a sequia. Se describe los aspectos relacionados con la selección de genotipos de interés, la multiplicación de los mismos mediante técnicas de cultivo in vitro y enraizamiento de estacas, la producción de plantas, el establecimiento de ensayos clonales y la evaluación inicial de los mismos.

Se evalúa el desempeño inicial de los clones en terreno y se discute las perspectivas y aplicaciones futuras de la propagación vegetativa y silvicultura clonal de $E$. camaldulensis.

Se concluye que la combinación de tecnologias de propagación asexual, particularmente organogénesis somática y enraizamiento de estacas, resulta apropiada para multiplicar individuos adultos de $E$. camaldulensis y que el desempeño inicial exhibido en terreno por clones selectos en función de su tolerancia a sequia, ofrece interesantes perspectivas para desarrollar cultivos forestales en las zonas semiáridas del pais

Palabras claves: Eucalyptus camaldulensis, clonación, resistencia a sequia

'Instituto Forestal. Sede Bio Bio Chile.bgutierr@infor.cl. mmolina@infor cl 


\section{Eucalyptus camaldulensis CLONES SELECTION, DEPLOYMENT AND EARLY EVALUATION FOR ARID AND SEMIARID ZONES}

\section{SUMMARY}

The arid and semi-arid zones in central Chile, are characterized by adverse climatic conditions, which limit the productive development of agricultural and silvicultural activities. Several efforts have been done to develop productive alternatives compatible with such limitation, identifying among them the culture of drought tolerant forest species.

Among such species. Eucalyptus camaldulensis has shown a great adaptation to drought conditions. At the present time, it is one of the best known alternatives to establish forest cultures in the semiarid regions. On the matter, the great extension of the natural distribution of $E$. camaldulensis in its original natural distribution confers it a considerable intraspecific variability in several interesting traits, including the drought tolerance. This variability, added to the empirical experience obtained in the establishment, management and evaluation of $E$. camaldulensis field trials, allows recognizing the existence of individual trees with a superior tolerance to water stress condition. which is able to exhibit interesting growth rates, still under detrimental water supplies conditions.

Recognizing the previous statements, the present article shows the main results of a research project aimed to the clonal spread of $E$. camaldulensis individuals, selected by their growth and drought resistance. Topics related with genotype selection, their multiplication through in vitro culture and rooting cuttings techniques, plant production. establishment of clonal trials and their initial evaluation are described.

The early field performance of the clones is analyzed. The perspectives and future uses of the vegetative propagation and $E$. camaldulensis clonal silviculture are discussed. It is concluded that the mix of vegetative propagation technologies, particularly somatic organogenesis and rooting cutting, are suitable to multiply E. camaldulensis adult trees. Furthermore, the early field performance exhibited by clones selected according to their drought tolerance, offers interesting perspective to develop cultures in the semi-arid zones of the country.

Key words: Eucalyptus camaldulensis, cloning, drought tolerance 


\section{INTRODUCCIÓN}

Las zonas áridas y semiáridas de la parte central de Chile, se caracterizan por condiciones climáticas especialmente adversas que limitan el desarrollo productivo del sector silvoagropecuario. Aún asi, existen alternativas productivas compatibles con tales limitaciones, pero que requieren de cierto desarrollo tecnológico antes de que puedan ser difundidas y adoptadas por el sector productivo.

Al respecto, existe concordancia en que la diversificación forestal con especies del género Eucalyptus, tales como $E$. camaldulensis y $E$. cladocalyx, representa una alternativa efectiva y viable, con especies que demuestran una adecuada adaptación a condiciones de aridez, caracteristica favorables de crecimiento y generación temprana de retornos económicos (8 a 12 años). A su vez, el cultivo de tales especies brinda interesantes oportunidades de comercialización y autoabastecimiento de madera a las comunidades agricolas, sociedades agricolas, productores vitivinicolas y fruticolas, permitiéndoles ampliar su base productiva, en particular en los periodos con escasa demanda de sus productos, o bien autoabastecerse de insumos como son las maderas redondas (polines, estacas, tirantes, postes, etc.).

En atención a estas consideraciones, el Instituto Forestal desarrolló el Proyecto FIA - BID: Masificación Clonal de Genotipos Forestales de Interés Comercial para la Zona Árida y Semi Árida del Pais, el cual fue ejecutado entre los años 2001 y 2005 en conjunto con las instituciones asociadas: Vivero Forestal Cavilolén, Sociedad Agricola y Ganadera El Tangue, Comunidad Agricola de Cuz Cuz y CONAF Coquimbo.

El objetivo de tal iniciativa fue aumentar la productividad de las plantaciones de Eucalyptus camaldulensis que se establezcan en las zonas semiáridas del pais, aspecto que metodológicamente se abordó mediante la combinación de elementos de mejoramiento genético y biotecnologias de propagación. En efecto, como primera medida, el proyecto seleccionó en sitios con acentuadas condiciones de aridez a individuos de $E$. camaldulensis que exhibian caracteristicas productivas superiores en tèrminos de volumen y forma, generando con ellos una base genética que posteriormente fue utilizada para implementar programas de multiplicación clonal.

A partir de tales individuos se desarrolló una metodologia operativa para la clonación de estos genotipos mediante técnicas de micro y macropropagación, que permitieron generar copias vegetativas que fueron establecidas en tres ensayos clonales.

Los aspectos operativos de estas actividades, asi como los resultados obtenidos de su aplicación, son discutidos en este articulo. En él se presenta las metodologias utilizadas, asi como los resultados obtenidos en las etapas de selección de genotipos superiores, protocolos de multiplicación vegetativa y desempeño inicial de los clones establecidos en ensayos de terreno. 


\section{MATERIAL Y METODO}

\section{Selección de Genotipos Superiores}

Fue implementado un sistema de selección indirecta para tolerancia a sequia, basado en la rigurosa individualización de árboles que, creciendo en zonas con severas restricciones hidricas, eran capaces de exhibir caracteristicas superiores de volumen y forma en relación a los restantes árboles que crecian bajo las mismas condiciones de sitio. Para estos efectos se prospectó ensayos y plantaciones de $E$. camaldulensis, haciendo uso del sistema de selección denominado de árboles de comparación, el cual resulta particularmente efectivo para realizar selección masal en rodales coetáneos.

\section{MICROPROPAGACIÓN}

Desde los árboles seleccionados se cosechó material vegetativo correspondiente a ramas con brotes del último periodo de crecimiento, este fue identificado, embalado y despachado a laboratorio para iniciar el proceso de micropropagación.

Una vez en laboratorio, el material fue sometido a un protocolo de desinfección mediante lavados, remojos y enjuagues con agua, detergentes, fungicidas e hipoclorito de sodio. Durante este proceso también se consideró la aplicación de una solución antioxidante compuesta por ácido citrico y ácido ascórbico en dosis de $500 \mathrm{mg} / \mathrm{l}$ de cada compuesto.

Desde el material desinfectado se extrajo segmentos nodales que fueron establecidos in vitro, en frascos de borosilicato ("snap" de $1 / 2$ pulgada de diametro) con el medio de cultivo MS (Murashige - Skoog). Los cultivos asi iniciados, fueron mantenidos por tres meses en sala de crecimiento, con una temperatura de $22+/-2^{\circ} \mathrm{C}$ y un fotoperiodo de 16 horas.

La multiplicación de brotes in vitro a partir de los explantes iniciales se extendio por 12 meses en medio MS reducido a la mitad y suplementado con hormonas del grupo de las citoquininas

Una vez multiplicados los brotes se procedió a elongarlos y enraizarlos para obtener vitroplantas. Para la elongación se utilizó el mismo medio que para la multiplicación de brotes, pero reduciendo la concentración de BAP (citoquinina). Alternativamente, en los clones que no responden a este procedimiento por si solo, se lo complementó con etiolación para conseguir que sus brotes se elonguen.

El enraizamiento se realizó en el medio de cultivo MS, con los macronutrientes reducidos en su concentración y complementado con la auxina AIB (ácido Indolbutírico). Para este efecto, los brotes fueron dispuestos durante siete dias en oscuridad, para luego ser mantenidos con un fotoperiodo de $16 \mathrm{~h} \mathrm{luz}$, a una temperatura de $22^{\circ} \mathrm{C}$, hasta la aparición y desarrollo de las raices (aproximadamente 18 dias), para luego iniciar la etapa de aclimatación.

La aclimatación de las vitroplantas, para acondicionarlas a condiciones ex vitro. fue realizada en dos etapas, inicialmente en laboratorio y posteriormente en invernadero. Primero las 
plantas fueron traspasadas en cámara de flujo laminar a cajas plásticas estériles que contenian un sustrato compuesto por turba y perlita y complementado con un medio nutritivo liquido (MS completo, sin sacarosa). Las cajas fueron cubiertas con una doble lámina de polietileno, removiéndola gradualmente a medida que la planta se adapta a condiciones ambientales normales. Una vez retirada completamente la cubierta, las plantas fueron trasladadas a invernadero y transplantadas a macetas de 3.5 litros de capacidad volumétrica, utilizando corteza de pino como sustrato, suplementada con fertilizantes de liberación controlada.

\section{MACROPROPAGACIÓN}

Una vez aclimatadas las plantas producidas por micropropagación, estas dieron origen a plantas madres. El manejo de las plantas madres consideró fundamentalmente la adecuada nutrición del seto y la aplicación de podas que le confirieran una estructura de copa compatible con la abundante producción de brotes para confeccionar estacas enraizables.

El periodo de permanencia en invernadero fue de aproximadamente 60 dias, momento a partir del cual se apreciaba una lignificación del tallo principal y un mayor desarrollo de la parte aérea de la plantas. Con posterioridad las plantas fueron traspasadas al vivero, con sombreadero durante la primera semana, y retiro paulatino de este, de modo de conseguir un endurecimiento de las plantas que les permitiera resistir la primera poda de formación.

Las podas tuvieron por objetivo suprimir el crecimiento apical y promover la proliferación de brotes laterales. Normalmente se aplicó un topping o poda apical para favorecer el crecimiento de 3 a 5 brotes laterales bien distribuidos. En estos brotes laterales eran colectadas las estacas con cortes sobre el primer par de hojas, de modo que de cada corte generará 2 brotes nuevos para una cosecha posterior. En lo sucesivo se fue extrayendo y favoreciendo brotes de modo de conformar una estructura achaparrada, con múltiples brotes y sin dominancia apical.

Previo a la definición de un protocolo operativo para el enraizamiento de estacas provenientes de plantas madres, se estableció un ensayo en vivero e invernadero, probando tres sustratos (corteza, corteza:perlita y turba:arena) y la aplicación de auxina (ácido indolbutirico. $5.000 \mathrm{ppm}$ ) sobre la supervivencia y el enraizamiento de las estacas.

El ensayo de enraizamiento consideró 9 tratamientos, estructurados en un diseño completamente aleatorizado, con tres repeticiones de 28 estacas por tratamiento. Los ensayos fueron evaluados 8 semanas después de ser establecidos.

La confección de las estacas de $E$. camaldulensis se realizó a partir de brotes extraidos desde los setos o plantas madres. A partir de estos brotes se seccionó segmentos de tallo de 6 a $8 \mathrm{~cm}$ de longitud con un par de hojas sanas y un diámetro no inferior a $2 \mathrm{~mm}$.

Las estacas confeccionadas en el paso anterior fueron sometidas a un tratamiento hormonal mediante el cual se le aplicaron auxinas exógenas para mejorar su respuesta rizogénica. Una vez aplicado el tratamiento hormonal, las estacas fueron insertadas en contenedores previamente preparados con el sustrato seleccionado, arena:turba en partes 
iguales. Los contenedores utilizados correspondieron a bandejas de poliestireno expandido (speedlings) de 84 cavidades con un volumen de sustrato por cavidad de $130 \mathrm{~cm}^{3}$.

El enraizamiento se verificó entre las 6 y 8 semanas, periodo durante el cual las estacas debieron permanecer bajo condiciones de invernadero. Durante este periodo se procuró mantener una humedad relativa alta (superior al 70\%) y una temperatura ambiental de 22 a $24^{\circ} \mathrm{C}$.

Una vez enraizadas, las estacas fueron manejadas en vivero como una planta convencional, hasta que alcanzaron el estado apropiado para ser despachada a terreno y establecer los ensayos clonales.

\section{ENSAYOS CLONALES}

Las plantas obtenidas mediante enraizamiento de estacas corresponden a copias vegetativas de los árboles superiores seleccionados por el proyecto. Éstas fueron establecidas en tres ensayos clonales, con el objeto de probar el desempeño de los clones en condiciones representativas de aquellas donde serán establecidos operacionalmente. Los ensayos fueron instalados en Pullally, Cuz Cuz y EI Tangue (Cuadro $N^{\circ} 1$ ).

\section{Cuadro $\mathrm{N}^{\circ} 1$ \\ UBICACIÓN DE LOS ENSAYOS CLONALES DE E. camaldulensis}

\begin{tabular}{|c|l|l|l|l|}
\hline REGIÓN & \multicolumn{1}{|c|}{ PROVINCIA } & \multicolumn{1}{c|}{ COMUNA } & \multicolumn{1}{c|}{ PREDIO } & \multicolumn{1}{c|}{ PROPIETARIO } \\
\hline Valparaiso & Petorca & La Ligua & Pullally & Comunidad Javier Estay y Otros \\
\hline Coquimbo & Choapa & Illapel & Cuz Cuz & Comunidad Agricola Cuz Cuz \\
\hline Coquimbo & Elqui & Tongoy & El Tangue & Sociedad Agricola y Ganadera El Tangue \\
\hline
\end{tabular}

\section{PULLALLY}

Este ensayo se ubica a aproximadamente $160 \mathrm{Km}$ al norte de Santiago, en la Comuna de La Ligua, V Región. Corresponde a una zona mediterránea con marcada influencia marina. La precipitación media anual es de $250 \mathrm{~mm}$ fuertemente concentrada en los meses invernales. La influencia costera se manifiesta en temperaturas templadas que no sobrepasan los $25^{\circ} \mathrm{C}$, con una muy baja incidencia de heladas. El suelo corresponde a arenas originadas en una duna fósil de baja fertilidad (Smith, 1997).

\section{CUZ-CUZ}

Los terrenos de la comunidad agricola de Cuz Cuz se enmarcan en el clima de Estepa Cálido que se caracteriza por la sequedad del aire y la ausencia de nubosidad. Sus temperaturas son mayores que en la costa, las precipitaciones son muy irregulares y escasas y los periodos de sequía son caracteristicamente extensos (8 meses o más). 
El sitio seleccionado para establecer el ensayo corresponde a la parte baja de una ladera orientada hacia el sur-este, con una acentuada pendiente de cerca del $90 \%$ y suelo severamente compactado.

\section{El Tangue}

Se encuentra en un clima clasificado como de estepa con nubosidad abundante. Se caracteriza por abundante nubosidad baja. La cercania del mar produce amplitudes térmicas bajas. Las precipitaciones presentan un régimen frontal, con máximos en el invierno (junio, julio y agosto) donde precipita cerca del $80 \%$ del total anual. En El Tangue caen 107 mm anuales.

El sitio seleccionado corresponde a un sector denominado El Tranque en el estero Bachingo, dentro de la hacienda "EI Tangue". Es una superficie plana de suelo liviano con la posibilidad de contar con agua para riegos de socorro en verano. (30 $\left.18^{\prime} \mathrm{S} 71^{\circ} 34^{\prime} \mathrm{O}\right)$

En los tres sitios anteriores se acondicionó el terreno para la plantación, mediante roce de la vegetación existente, aradura y subsolado del suelo y construcción de cerco perimetral de protección contra animales silvestres y ganado.

La plantación fue manual, a un espaciamiento de $3 \times 3$ metros y de acuerdo a un diseño previamente definido. En todos los sitios se usó un diseño compuesto por 4 bloques completos al azar, donde cada clon era representado por una parcela lineal de 4 rametos. En total cada clon comprende 16 plantas en cada ensayo.

El numero e identificación de los clones considerados en cada ensayo se presenta en el Cuadro $N^{\circ} 2$.

La plantación propiamente tal se efectuó durante el mes de octubre de 2005, y en la misma ocasión se aplicó gel, fertilizante y un riego inicial. La primera medición, que es la que se analiza en este trabajo, corresponde a la efectuada en agosto de 2006, a los 10 meses de edad.

\section{Cuadro $\mathrm{N}^{\circ} 2$}

MATERIAL GENÉTICO CONSIDERADO EN LOS ENSAYOS CLONALES DE E. camaldulensis

\begin{tabular}{|c|l|c|c|c|}
\hline $\begin{array}{c}\text { Código } \\
\text { Clon }\end{array}$ & \multicolumn{1}{|c|}{$\begin{array}{c}\text { Identificación } \\
\text { de Terreno }\end{array}$} & Pullally & Cuz Cuz & El Tangue \\
\hline $\mathbf{1}$ & $\begin{array}{l}\text { Ensayo Tantehue. } \\
\text { Bq 1; proced 2, Prog 16; Arbol 3 }\end{array}$ & $\mathrm{X}$ & $\mathrm{X}$ & $\mathrm{X}$ \\
\hline $\mathbf{3}$ & $\begin{array}{l}\text { Ensayo Tantehue. } \\
\text { Bq 1; proced 9. Prog 98; Arbol 3 }\end{array}$ & $\mathrm{X}$ & $\mathrm{X}$ & $\mathrm{X}$ \\
\hline $\mathbf{5}$ & $\begin{array}{l}\text { Ensayo Tantehue. } \\
\text { Bq 2; proced 2, Prog 9; Arbol 1 }\end{array}$ & $\mathrm{X}$ & $\mathrm{X}$ & \\
\hline $\mathbf{6}$ & $\begin{array}{l}\text { Ensayo Tantehue. } \\
\text { Bq 2: proced 8, Prog 87; Arbol 3 }\end{array}$ & & \\
\hline
\end{tabular}




\begin{tabular}{|c|c|c|c|c|}
\hline $\begin{array}{l}\text { Código } \\
\text { Clon }\end{array}$ & $\begin{array}{l}\text { Identificación } \\
\text { de Terreno }\end{array}$ & Pullally & Cuz Cuz & El Tangue \\
\hline 9 & $\begin{array}{l}\text { Ensayo Tantehue. } \\
\text { Bq 3; proced 6, Prog 63; Arbol } 1\end{array}$ & $x$ & $x$ & $x$ \\
\hline 11 & $\begin{array}{l}\text { Ensayo Tantehue. } \\
\text { Bq 4; proced 6, Prog 60; Arbol } 1\end{array}$ & $x$ & $x$ & $x$ \\
\hline 21 & $\begin{array}{l}\text { Ensayo Tantehue. } \\
\text { Bq 8; proced 3, Prog 22; Arbol } 4\end{array}$ & $x$ & $x$ & \\
\hline 27 & $\begin{array}{l}\text { Ensayo Tantehue. } \\
\text { Bq 9; proced 2, Prog 19; Arbol } 2\end{array}$ & $x$ & & $x$ \\
\hline 29 & $\begin{array}{l}\text { Ensayo Tantehue. } \\
\text { Bq 10; proced 5. Prog 52; Arbol } 1\end{array}$ & $x$ & $x$ & $x$ \\
\hline 31 & $\begin{array}{l}\text { Ensayo Tantehue. } \\
\text { Bq 10: proced 8, Prog 81: Arbol } 4\end{array}$ & $x$ & $x$ & $x$ \\
\hline 43 & $\begin{array}{l}\text { Ensayo Longotoma } \\
32^{\circ} 24,665^{\prime}: 71^{\circ} 21,724^{\circ}\end{array}$ & $x$ & $x$ & $x$ \\
\hline 44 & $\begin{array}{l}\text { Ensayo Longotoma } \\
32^{\circ} 24,676^{\prime}: 71^{\circ} 20,670^{\prime}\end{array}$ & $x$ & & $x$ \\
\hline 50 & Plantación masiva Tantehue & $x$ & $x$ & $x$ \\
\hline
\end{tabular}

\section{RESULTADOS}

\section{Selección de Genotipos Superiores}

Como resultado del proceso de selección de árboles plus se identificaron 62 individuos de $E$. camaldulensis, distribuidos en 8 rodales. Desde 45 de tales árboles se extrajeron propágulos vegetativos para iniciar cultivos in vitro, 37 de los cuales superaron la etapa de desinfección y permitieron establecer explantes in vitro libres de contaminación.

\section{Micropropagación}

Los ensayos de desinfección y transferencia del tejido a cultivo in vitro permitieron obtener un porcentaje medio de contaminación del $22 \%$, con una gran variación entre clones $(1,7 \%$ a $80 \%)$. La mortalidad por concepto de oxidación de explantes alcanzó un promedio de $41,7 \%$.

La multiplicación de brotes tambièn tuvo una marcada variación entre clones, obteniéndose un promedio de 32 explantes por clon.

Durante la etapa de elongación de brotes se observó una respuesta muy diferenciada en función de los clones utilizados, de modo que sólo en 13 de ellos se logró inducir una elongación compatible con la etapa de enraizamiento posterior.

El protocolo de enraizamiento comentado en la metodologia permitió obtener un $100 \%$ de éxito en la inducción de rizogénesis adventicia, observándose que el principal factor que influye en la eficiencia de esta metodologia es el hecho de aplicarla sobre brotes suficientemente 
elongados.

La metodologia de aclimatación permitió obtener una proporción de vitroplantas aclimatadas a condiciones ex vitro del orden del 100\%. Esta alta tasa de sobrevivencia al extraer las plantas desde los frascos de cultivo se mantiene en invernadero y vivero.

Las plantas micropropagadas permitieron conformar un Banco de Plantas Madres que fue utilizado para desarrollar e implementar los procedimientos de macropropagación mediante enraizamiento de estacas y de esta forma incrementar el número de réplicas vegetativas de cada clon seleccionado, hasta contar con rametos suficientes para establecerlos en los respectivos ensayos clonales.

\section{Macropropagación}

Los resultados de los ensayos de enraizamiento de estacas descritos en la metodologia se resumen en el Cuadro $\mathrm{N}^{\circ} 3$. Los resultados derivados del análisis de varianza, efectuados sobre los datos transformados en Unidades de Bliss, indican la existencia de diferencias estadisticamente significativas entre tratamientos, tanto para la supervivencia como para el enraizamiento (alfa $=0,05$ ). Esta situación permite conformar grupos de tratamientos que estadisticamente alcanzan niveles de enraizamiento similares, los que de acuerdo con la dócima de Duncan se representan con letras iguales en el cuadro $\mathrm{N}^{\circ} 3$.

\section{Cuadro $\mathrm{N}^{\circ} 3$}

SUPERVIVENCIA Y ENRAIZAMIENTO A LAS 8 SEMANAS DE ESTACAS DE E. camaldulensis (PORCENTAJE PROMEDIO POR TRATAMIENTO)

\begin{tabular}{|c|c|c|c|c|c|}
\hline \multicolumn{3}{|c|}{ Descripción del Tratamiento } & \multirow{2}{*}{$\begin{array}{c}\text { Tratamiento } \\
\text { T0 }\end{array}$} & \multirow{2}{*}{$\begin{array}{c}\begin{array}{c}\text { Supervivencia } \\
(\%)\end{array} \\
29.8\end{array}$} & \multirow{2}{*}{$\begin{array}{c}\begin{array}{c}\text { Enraizamiento } \\
(\%)\end{array} \\
25,0 \mathrm{~b}\end{array}$} \\
\hline \multirow{6}{*}{ Invernadero } & \multirow{2}{*}{ Corteza } & $\operatorname{Sin} A \mid B$ & & & \\
\hline & & AIB $5.000 \mathrm{ppm}$ & T1 & 33,3 & $29.8 \mathrm{~b}$ \\
\hline & \multirow{2}{*}{ Corteza Perlita } & $\operatorname{Sin} A I B$ & $\mathrm{~T} 2$ & 19.0 & $16.7 \mathrm{ab}$ \\
\hline & & AIB $5.000 \mathrm{ppm}$ & $\mathrm{T} 3$ & 38,1 & $34,5 \mathrm{bc}$ \\
\hline & \multirow{2}{*}{ Arena Turba } & $\operatorname{Sin} A \mid B$ & T4 & 23,8 & $21,4 \quad b$ \\
\hline & & AIB $5.000 \mathrm{ppm}$ & T5 & 61,9 & $51,2 \mathrm{c}$ \\
\hline \multirow{3}{*}{ Vivero } & Corteza & $\operatorname{Sin} A \mid B$ & T6 & 31,0 & 7.1 a \\
\hline & Corteza:Perlita & AIB $5.000 \mathrm{ppm}$ & $\mathrm{T7}$ & 14.3 & 4,8 a \\
\hline & Arena: Turba & AIB $5.000 \mathrm{ppm}$ & T8 & 65.5 & $22,6 \quad b$ \\
\hline \multicolumn{4}{|c|}{ Totales } & 35,2 & 23,7 \\
\hline
\end{tabular}


E. camaldulensis es una especie relativamente fácil de enraizar. Experiencias desarrolladas por Awad y Gutiérrez (1997) señalan porcentajes de enraizamiento entre 65 y $85 \%$, valores superiores a los alcanzados en este estudio. Los autores anteriores (op cit) concluyen que no existe efecto del sustrato ni de la aplicación de auxina sobre el enraizamiento. pero que si hay un efecto clonal que determina la respuesta rizogénica. Sin embargo en este estudio se observan diferencias estadisticamente significativas entre tratamientos. destacándose a la aplicación de auxina, el uso de turba con arena y al enraizamiento bajo invernadero como las condiciones más apropiadas para inducir la formación de raices adventicias

Al respecto, los menores enraizamientos obtenidos en este estudio pueden ser atribuidos a ese efecto clonal, asi como también al distinto origen de las plantas madres utilizadas como fuentes de estacas. En el estudio mencionado se utilizaron plantas juveniles provenientes de semilla, mientras que en este nuevo ensayo se usaron réplicas micropropagadas de sólo un clon adulto, las que si bien exhibian un grado importante de rejuvenecimiento, correspondian a plantas con escasa lignificación y de brotes más suculentos. Lo mismo puede argumentarse para explicar las diferencias en el efecto de la aplicación de auxinas.

En cualquier caso, el ensayo permite confirmar que en especies fáciles de enraizar, el control de las variables ambientales durante el enraizamiento se hace menos critico que en especies de arraigamiento más complicado. Efectivamente, en las precarias condiciones de las estacas manejadas en vivero se obtuvo un enraizamiento promedio, que si bien es inferior al obtenido en invernadero, presenta tratamientos, como el T8, que no se diferencia estadisticamente de otros establecidos dentro del invernadero y que incluso supera a algunos de estos.

No obstante, la calidad de las raices y de los brotes producidos se hace muy diferente en ambas condiciones. En vivero las estacas presentan un muy limitado desarrollo de brotes y sus hojas se presentan coriáceas, gruesas y rojizas. Por el contrario, en invernadero el desarrollo de los brotes es muy rápido, estos se elongan con facilidad y se presentan verdes y muy turgentes.

En invernadero se observa una alta correlación entre sobrevivencia y enraizamiento. situación que no se observa en las estacas de vivero. En esta última condición, las estacas si bien se pueden mantener vivas, enraízan en una proporción mucho menor, observándose una gran cantidad de ellas que sólo exhiben un abundante callo basal, que no se ha diferenciado en raíces. Este fenómeno se observa en forma muy escasa en las estacas manejadas en invernadero, donde casi la totalidad de las estacas vivas exhiben formación de raices.

\section{Ensayos Clonales}

La sobrevivencia de los ensayos clonales fue claramente inferior a la observada en ensayos con plantas de semillas de E. camaldulensis (Gutiérrez y Chung, 1993). Sin embargo, esta diferencia parece obedecer a las condiciones más favorables en que se establecieron los ensayos con plantas de semilla. Al comparar la sobrevivencia del ensayo clonal Pullally 
$(91,3 \%)$ con la reportada para un ensayo establecido en el mismo sitio con plantas obtenidas sexualmente, se obtienen resultados muy similares.

En términos de crecimiento inicial, los clones se comparan muy favorablemente con plantas obtenidas por propagación sexual. En efecto, a los 10 meses de edad los ensayos clonales presentaron una altura media de $1,2 \mathrm{~m}$ y un diámetro de cuello (DAC) de $13,9 \mathrm{~mm}$, mientras que plantas de semilla, establecidas en un sitio equivalente al de Pullally (sector Longotoma), exhibian a los dos años de edad una altura media de $1,16 \mathrm{~m}$ y un DAC medio de $18 \mathrm{~mm}$ (Gutiérrez y Chung, 1993).

El comportamiento general de los clones representados en los ensayos, en términos de supervivencia y crecimiento inicial en DAC y altura, después de 10 meses de desempeño en terreno se resume en el Cuadro $\mathrm{N}^{\circ} 4$. Posteriormente, en los Cuadros $\mathrm{N}^{\circ} 5$ al 7 se describe el comportamiento exhibido por los clones en cada uno de los sitios ensayados.

\section{Cuadro $\mathrm{N}^{\circ} 4$}

DESEMPEÑO INICIAL DE CLONES DE E. camaldulensis ESTABLECIDOS EN TRES SITIOS DE LA IV Y V REGIONES DE CHILE, EVALUADO A LOS 10 MESES DE EDAD

\begin{tabular}{|c|c|c|c|c|c|}
\hline $\begin{array}{l}\text { Código } \\
\text { clon }\end{array}$ & $\begin{array}{c}\mathbf{N}^{\circ} \text { de } \\
\text { plantas } \\
\text { establecidas }\end{array}$ & $\begin{array}{l}\mathrm{N}^{\circ} \mathrm{de} \\
\text { plantas } \\
\text { vivas }\end{array}$ & $\begin{array}{c}\text { Supervivencia } \\
(\%)\end{array}$ & $\begin{array}{c}\text { DAC } \\
\text { Promedio } \\
(\mathrm{mm})\end{array}$ & $\begin{array}{c}\text { Altura } \\
\text { Promedio } \\
\text { (m) }\end{array}$ \\
\hline 1 & 100 & 63 & 63.0 & 13.7 & 1,2 \\
\hline 3 & 83 & 50 & 60,2 & 13.2 & 1.3 \\
\hline 5 & 46 & 21 & 45.7 & 12.8 & 1,1 \\
\hline 6 & 16 & 8 & 50.0 & 15,8 & 1,4 \\
\hline 9 & 39 & 28 & 71.8 & 17,1 & 1,6 \\
\hline 11 & 56 & 26 & 46,4 & 12,4 & 1,1 \\
\hline 21 & 12 & 5 & 41.7 & 15,8 & 1.4 \\
\hline 27 & 22 & 3 & 13.6 & 13.7 & 1.1 \\
\hline 29 & 56 & 32 & 57,1 & 14,8 & 1.2 \\
\hline 31 & 86 & 50 & 58,1 & 14.5 & 1.4 \\
\hline 43 & 100 & 56 & 56,0 & 13,8 & 1.0 \\
\hline 44 & 16 & 2 & 12,5 & 12.5 & 0.8 \\
\hline 50 & 60 & 30 & 50.0 & 12.5 & 1,2 \\
\hline Total & 692 & 374 & 54,0 & 13,9 & 1,2 \\
\hline
\end{tabular}




\section{Cuadro $\mathrm{N}^{\circ} 5$}

DESEMPEÑO INICIAL DE CLONES DE E. camaldulensis ESTABLECIDOS EN EL ENSAYO CUZ CUZ

\begin{tabular}{|c|c|c|c|c|c|}
\hline $\begin{array}{l}\text { Código } \\
\text { clon }\end{array}$ & $\begin{array}{c}\mathbf{N}^{\circ} \text { de } \\
\text { plantas } \\
\text { establecidas }\end{array}$ & $\begin{array}{l}\mathrm{N}^{\circ} \mathrm{de} \\
\text { plantas } \\
\text { vivas }\end{array}$ & $\begin{array}{c}\text { Supervivencia } \\
(\%)\end{array}$ & $\begin{array}{c}\text { DAC } \\
\text { Promedio } \\
(\mathrm{mm})\end{array}$ & $\begin{array}{c}\text { Altura } \\
\text { Promedio } \\
\text { (m) }\end{array}$ \\
\hline 1 & 32 & 22 & 68,8 & 18.5 & 1,4 \\
\hline 3 & 16 & 14 & 87.5 & 18,4 & 1.7 \\
\hline 5 & 16 & 2 & 12,5 & 19.5 & 1.3 \\
\hline 6 & 16 & 8 & 50,0 & 15,8 & 1,4 \\
\hline 9 & 16 & 12 & 75,0 & 19.5 & 1,8 \\
\hline 11 & 16 & 5 & 31,3 & 20,2 & 1,8 \\
\hline 21 & 12 & 5 & 41,7 & 15.8 & 1,4 \\
\hline 27 & 10 & 1 & 10,0 & 17,0 & 1,6 \\
\hline 29 & 16 & 8 & 50.0 & 17.9 & 1,5 \\
\hline 31 & 26 & 18 & 69,2 & 19,3 & 1,7 \\
\hline 43 & 20 & 18 & 90,0 & 18,3 & 1,2 \\
\hline 44 & 12 & 0 & 0,0 & .. & .. \\
\hline 50 & 16 & 5 & 31,3 & 18,8 & 1,6 \\
\hline Total & 224 & 118 & 52,7 & 18,4 & 1,5 \\
\hline
\end{tabular}

Cuadro $N^{\circ} 6$

DESEMPEÑO INICIAL DE CLONES DE E. camaldulensis ESTABLECIDOS EN EL ENSAYO

PULLALLY

\begin{tabular}{|c|c|c|c|c|c|}
\hline $\begin{array}{c}\text { Código } \\
\text { clon }\end{array}$ & $\begin{array}{c}\mathbf{N}^{\circ} \text { de } \\
\text { plantas } \\
\text { establecidas }\end{array}$ & $\begin{array}{c}\mathbf{N}^{\circ} \text { de } \\
\text { plantas } \\
\text { vivas }\end{array}$ & $\begin{array}{c}\text { Supervivencia } \\
\mathbf{( \% )}\end{array}$ & $\begin{array}{c}\text { DAC } \\
\text { Promedio } \\
\text { (mm) }\end{array}$ & $\begin{array}{c}\text { Altura } \\
\text { Promedio } \\
\text { (m) }\end{array}$ \\
\hline $\mathbf{1}$ & 32 & 32 & 100,0 & 12,0 & 1,1 \\
\hline $\mathbf{3}$ & 16 & 14 & 87,5 & 12,6 & 1,2 \\
\hline $\mathbf{5}$ & 16 & 14 & 87,5 & 12,2 & 1,1 \\
\hline $\mathbf{9}$ & 16 & 14 & 87,5 & 16,0 & 1,5 \\
\hline $\mathbf{1 1}$ & 16 & 14 & 87,5 & 11,1 & 1,1 \\
\hline $\mathbf{2 9}$ & 16 & 16 & 100,0 & 15,2 & 1,2 \\
\hline $\mathbf{3 1}$ & 16 & 14 & 87,5 & 13,5 & 1,4 \\
\hline $\mathbf{4 3}$ & 16 & 14 & 87,5 & 15,0 & 1,1 \\
\hline $\mathbf{5 0}$ & 16 & 14 & 87,5 & 10,8 & 1,1 \\
\hline Total & 160 & 146 & 91,3 & 13,0 & 1,2 \\
\hline
\end{tabular}




\section{Cuadro $\mathrm{N}^{\circ} 7$}

DESEMPEÑO INICIAL DE CLONES DE E. camaldulensis ESTABLECIDOS EN EL ENSAYO EL TANGUE

\begin{tabular}{|c|c|c|c|c|c|}
\hline $\begin{array}{c}\text { Código } \\
\text { clon }\end{array}$ & $\begin{array}{c}\mathbf{N}^{\circ} \text { de } \\
\text { plantas } \\
\text { establecidas }\end{array}$ & $\begin{array}{c}\mathbf{N}^{\circ} \text { de } \\
\text { plantas } \\
\text { vivas }\end{array}$ & $\begin{array}{c}\text { Supervivencia } \\
(\mathbf{\%})\end{array}$ & $\begin{array}{c}\text { DAC } \\
\text { Promedio } \\
(\mathbf{m m})\end{array}$ & $\begin{array}{c}\text { Altura } \\
\text { Promedio } \\
\text { (m) }\end{array}$ \\
\hline $\mathbf{1}$ & 36 & 9 & 25,0 & 9,1 & 0.7 \\
\hline $\mathbf{3}$ & 51 & 22 & 43,1 & 10,3 & 0,9 \\
\hline $\mathbf{5}$ & 14 & 5 & 35,7 & 11,8 & 0,8 \\
\hline $\mathbf{9}$ & $\mathbf{7}$ & 2 & 28,6 & 10,0 & 0,8 \\
\hline $\mathbf{1 1}$ & 24 & 7 & 29,2 & 9,4 & 0,7 \\
\hline $\mathbf{2 7}$ & 12 & 2 & 16,7 & 12,0 & 0,8 \\
\hline $\mathbf{2 9}$ & 24 & 8 & 33,3 & 11,0 & 0,8 \\
\hline $\mathbf{3 1}$ & 44 & 18 & 40,9 & 10,6 & 0,9 \\
\hline $\mathbf{4 3}$ & 64 & 24 & 37,5 & 10,0 & 0,7 \\
\hline $\mathbf{4 4}$ & 4 & 2 & 50,0 & 12,5 & 0,8 \\
\hline $\mathbf{5 0}$ & 28 & 11 & 39,3 & 11,9 & 0,9 \\
\hline Total & 308 & 110 & 35,7 & 10,5 & 0,8 \\
\hline
\end{tabular}

La mayor tasa de supervivencia $(91,3 \%)$ se obtuvo en el ensayo Pullally, mientras que el mayor crecimiento se logró en el ensayo Cuz Cuz. En el ensayo de El Tangue, tanto las variables de crecimiento como la supervivencia, exhiben sus valores más bajos.

El clon con mejor desempeño promedio en los tres ensayos corresponde al $\mathrm{N}^{\circ} 9$, que exhibe simultáneamente los valores más altos de supervivencia, altura y DAC. A nivel de ensayos individuales se observa cierta variación; en Cuz Cuz este mismo clon vuelve a ser el más eficiente, mientras que en EI Tangue su desempeño no supera al promedio del ensayo en ninguna de las tres variables evaluadas. Por su parte, en Pullally muestra los mejores valores de crecimiento, pero su supervivencia es inferior al promedio del ensayo.

El menor desempeño promedio lo exhibe el clon $N^{\circ} 44$, el cual fue representado sólo en dos ensayos, en Cuz Cuz experimentó una mortalidad completa, mientras que en el Tangue su supervivencia y crecimiento en DAC fueron los mejores del ensayo, siendo su crecimiento en altura intermedio entre el de los restantes clones evaluados en este sitio.

La variación observada sugiere la existencia a nivel inicial de efectos de interacción entre los clones y los sitios de plantación (interacción genotipo ambiente). En el Cuadro $\mathrm{N}^{\circ} 8$ se confirma esta situación para la variable altura, la cual exhibe bajos niveles de correlación entre los sitios evaluados, siendo las diferencias más acentuadas las que se presentan entre Pullally y Cuz Cuz. En el caso del DAC las correlaciones son mayores, indicando una menor incidencia de esta interacción genotipo ambiente. Respecto a la supervivencia, las mayores diferencias (menores valores de correlación) se observan entre los sitios de EI Tangue y Pullally, mientras 
que las correlaciones restantes son relativamente altas.

\section{Cuadro $\mathrm{N}^{\circ} 8$ \\ MATRIZ DE CORRELACIÓN ENTRE VARIABLES REPRESENTATIVAS DEL DESEMPEÑO DE CLONES DE E. camaldulensis}

\begin{tabular}{|c|c|c|c|c|c|c|c|c|c|}
\hline & \multicolumn{3}{|c|}{ Sobrevivencia } & \multicolumn{3}{|c|}{ DAC } & \multicolumn{3}{|c|}{ Altura } \\
\hline & $\begin{array}{l}\text { Cuz } \\
\text { Cuz }\end{array}$ & $\begin{array}{l}\text { El } \\
\text { Tangue }\end{array}$ & Pullally & $\begin{array}{l}\text { Cuz } \\
\text { Cuz }\end{array}$ & $\begin{array}{l}\text { EI } \\
\text { Tangue }\end{array}$ & Pullally & $\begin{array}{l}\text { Cuz } \\
\text { Cuz }\end{array}$ & $\begin{array}{l}\text { El } \\
\text { Tangue }\end{array}$ & Pullally \\
\hline \begin{tabular}{|l|} 
Cuz Cuz \\
El Tangue \\
Pullally \\
\end{tabular} & 1 & $\begin{array}{c}0,72 \\
1\end{array}$ & $\begin{array}{c}0,91 \\
0,16 \\
1 \\
\end{array}$ & & & & & & \\
\hline \begin{tabular}{|l|} 
Cuz Cuz \\
EI Tangue \\
Pullally \\
\end{tabular} & & & & 1 & $\begin{array}{c}0,82 \\
1\end{array}$ & $\begin{array}{c}0,41 \\
0,73 \\
1 \\
\end{array}$ & & & \\
\hline \begin{tabular}{|l|} 
Cuz Cuz \\
EI Tangue \\
Pullally \\
\end{tabular} & & & & & & & 1 & $\begin{array}{c}0,41 \\
1\end{array}$ & $\begin{array}{c}0,09 \\
0,25 \\
1 \\
\end{array}$ \\
\hline
\end{tabular}

\section{CONCLUSIONES}

Los procedimientos empleados en el proyecto para clonar a individuos selectos de $E$. camaldulensis resultaron apropiados para producir plantas de esta especie, aún cuando las tasa de enraizamiento de estacas resultaron inferiores a las señaladas en trabajos anteriores (Awad y Gutiérrez, 1997).

El desempeño de tales plantas en terreno es inicialmente mejor que el observado en plantas de semillas. No obstante, la evaluación efectuada resulta demasiado inicial para establecer comparaciones válidas entre clones.

Los valores de supervivencia de los ensayos clonales resultaron en general inferiores a los reportados para plantas de semillas. Esta diferencia parece obedecer a las condiciones ambientales de los sitios de plantación más que al origen vegetativo de las plantas. A este respecto, la época de plantación no fue la adecuada para la zona de ensayo y es esperable que un establecimiento en la época de mayor probabilidad de precipitaciones resulte más favorable.

\section{REFERENCIAS}

Awad, G. y Gutiérrez, B. 1997. Evaluación de la Capacidad Rizogénica de Progenies Hibridas F1 de Eucalyptus camaldulensis X E. globulus. Chile Forestal N²47, Marzo de 1997. 
Gutiérrez, B. y Chung, P. 1993. Crecimiento inicial de 23 procedencias y 196 familias de Eucalyptus camaldulensis Denh en cuatro sitios de la zona central de Chile. En: Ciencia e Investigacion Forestal 7(1): 5-22.

Gutiérrez B.; Ortiz O.; Molina M.; Chung, P.; Koch L.; González, M.; Casanova, K. y Soto H. 2005. Protocolos de Clonación para Eucalyptus camaldulensis: Macro y Micropropagación. Proyecto FIA: BIDPI-C-2001-1-F-050. Masificación Clonal de Genotipos Forestales de Interés Comercial para la Zona Árida y Semiárida del Pais. Instituto Forestal, Concepción, Chile. 62 p.

Smith, N. 1997. Comportamiento de Eucalyptus camaldulensis en Longotoma. Chile. Base para el mejoramiento de la especie en la zona semiárida. En: Valdebenito, G y Benedetti. S. (editores). Forestación y Silvicultura en Zonas Áridas y Semiáridas de Chile. INFOR-CORFO. Santiago, Chile. Pp: 186-196. 